\title{
Immunoperoxidase study of the secretory immunoglobulin system in colonic neoplasia
}

\author{
PETER ISAACSON \\ From the Department of Pathology, Southampton University Medical School, Southampton SO9 $4 X Y$
}

SUMMARY The relation of the secretory immunoglobulin system in the colon to colorectal cancer and dysplasia has been examined by staining routine formalin-fixed, paraffin-embedded sections from cases of carcinoma, adenoma and ulcerative colitis for secretory component (SC), IgA and J chain. In carcinomas there was a close relation between SC synthesis and differentiation and a similar relation was apparent between SC synthesis and degrees of dysplasia in adenomas. In both morphological and functional (SC synthesis) terms degrees of dysplasia in adenomas resembled degrees of differentiation in carcinomas suggesting that the essential "switch" in the progression towards neoplasia may occur at the level of the adenoma and that invasive malignancy can arise from dysplastic mucosa of varying severity. Actively regenerating mucosa in ulcerative colitis (UC) showed intense staining for SC as opposed to foci of precancerous dysplasia where, with one exception, staining was markedly reduced or absent, suggesting staining for SC could be useful in identifying foci of precancerous dysplasia in UC. In the absence of severe chronic inflammation, as in UC, the number of IgA-containing plasma cells was closely related to SC staining of neoplastic mucosa suggesting that SC may be important in the mechanism by which IgA lymphocytes home to the lamina propria of the colon.

Immunohistochemistry, by permitting histological observations relating structure and function, has considerably widened the scope of diagnostic and investigative histopathology and the development of the immunoperoxidase technique has meant that the pathologist can now study material in the setting with which he is most familiar and use stored material for retrospective studies. Not surprisingly, when immunoperoxidase techniques first became widely available, histopathologists immediately began to search for reliable immunohistochemical markers that were indicative of malignant transformation. One such serological marker, carcinoembryonic antigen (CEA), was already at hand and first reports of its value as an immunohistochemical marker of malignant change in colonic mucosa were encouraging. ${ }^{1}$ Subsequently, however, considerable doubt was cast over the value of CEA as a tissue or serological marker of malignancy $^{2}$ and the fate of many other immunohistochemical markers of malignancy has been similar. ${ }^{3}$ A more logical approach to immunohistochemical Accepted for publication 27 May 1981 changes in malignancy might be to attempt to demonstrate alterations in complex functions that are carried out by normal cells and tissues. In the intestine the secretory immunoglobulin system would seem to be an ideal set of functions to study in this way since it is well characterised ${ }^{4}$ and specific antisera to the three principal components of this system are available.

Immunoglobulin $\mathbf{A}(\operatorname{Ig} \mathbf{A})$ is secreted by intestinal lamina propria plasma cells as a dimer, the two molecules being linked by $\mathbf{J}$ (joining) chain. The dimeric $\operatorname{IgA}$ becomes attached to secretory component (SC) which is synthesised by mucosal epithelial cells and the IgA-J-SC complex is then transported to the lumenal surface of the gut mucosa through the mucosal cells. This paper reports the results of the immunohistochemical demonstration of the three components of the secretory immunoglobulin system (SC, IgA and $J$ chain) in normal rectal mucosa, in adenomas and in carcinomas and the application of these results to the characterisation and diagnosis of premalignant change as it occurs in ulcerative colitis. 

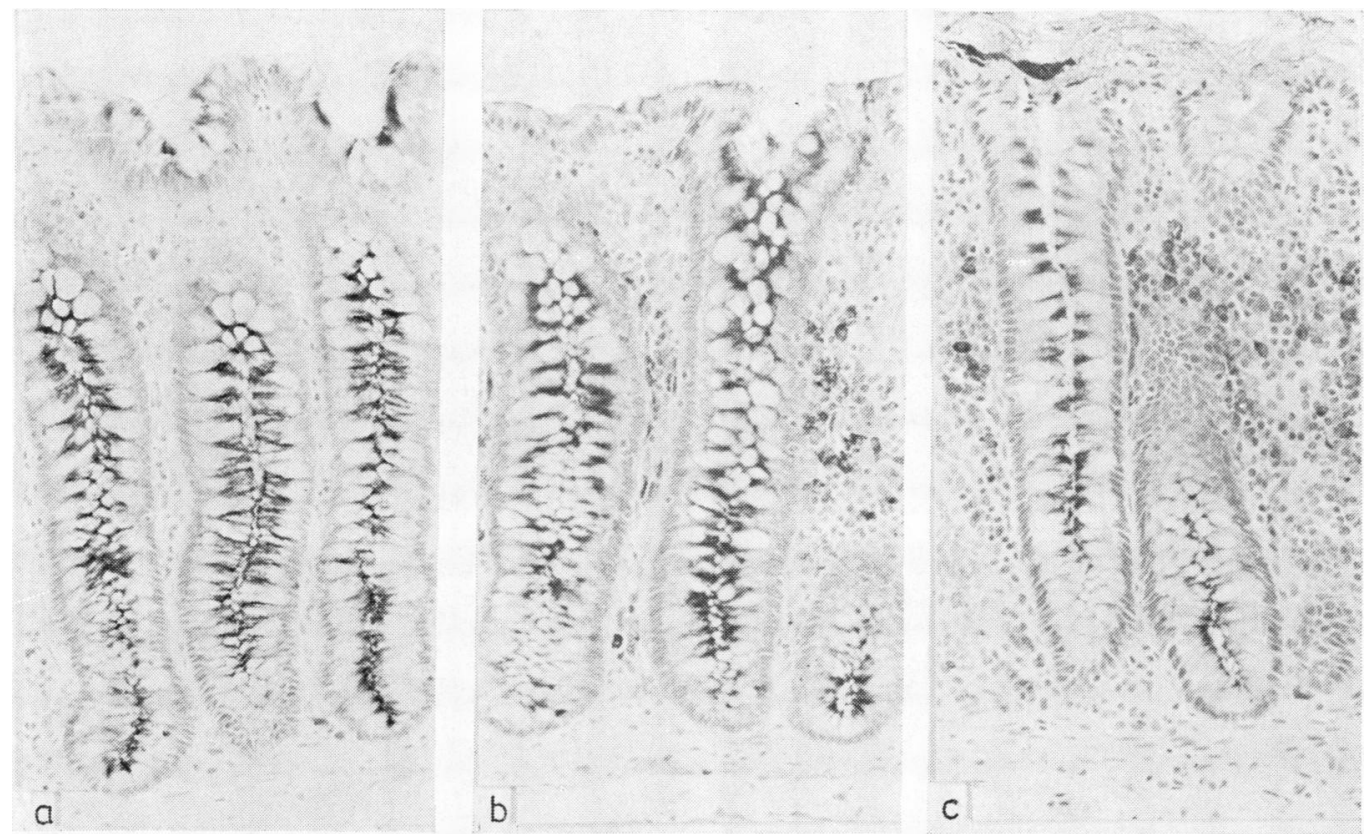

Fig. 1 Normal colonic mucosa stained for (a) SC, (b) IgA and (c) J chain. Immunoperoxidase $\times 100$.

\section{Material and methods}

All the sections studied were from formalin-fixed paraffin-embedded tissue removed either at laparotomy or endoscopically. Sections of normal colorectal mucosa were used to establish appropriate titres of antisera and as a base line for comparison with diseased mucosa. These sections were taken from colectomy specimens well away from the site of tumour or other localised disease-for example, diverticular disease. Adjacent normal mucosa was also included in most of the other sections studied. These included 25 cases ( 30 sections) of adenocarcinoma and 25 cases (52 sections) of adenoma (12 tubular adenomas, 9 tubovillous adenomas, 4 villous adenomas). In addition, 16 cases of ulcerative colitis were studied in eight of which precancerous dysplasia had been diagnosed. From these 16 cases 20 sections of inflamed and regenerative mucosa were selected for study and compared to 14 sections showing precancerous dysplasia.

Sections were stained by the PAP technique following trypsin digestion for $\operatorname{IgA}, \mathrm{J}$ chain and $\mathrm{SC}$. The details of the method used, sources of antisera and specificity controls have been previously described. ${ }^{5-7}$ In all cases adjacent sections were stained routinely with haematoxylin and eosin permitting detailed comparison between conventional hıstology and immunohistochemistry.

\section{Results}

NORMAL COLORECTAL MUCOSA (Fig. 1)

Staining for SC was strongly positive along the lateral membranes of mucosal cells and along the apical border of the crypts. No staining of mucin goblets was evident but cytoplasmic staining of non-mucin-containing columnar cells was present. The distribution of IgA and $J$ chain matched that of SC exactly with the exception that numerous plasma cells in the lamina propria showed strong cytoplasmic staining for $\operatorname{IgA}$ and rather weaker staining for $\mathbf{J}$ chain.

Relation of SC staining to severity of dysplasia (differentiation)

\begin{tabular}{|c|c|c|c|}
\hline$S C$ staining & $\begin{array}{l}\text { Mild dysplasia } \\
\text { (well differ'd) }\end{array}$ & $\begin{array}{l}\text { Mod dysplasia } \\
\text { (mod differ'd) }\end{array}$ & $\begin{array}{l}\text { Severe dysplasia } \\
\text { (poorly differ'd) }\end{array}$ \\
\hline \multicolumn{4}{|c|}{ Carcinoma 25 cases } \\
\hline normal & 6 & 1 & - \\
\hline reduced & 2 & 16 & 1 \\
\hline weak/absent & - & 一 & 20 \\
\hline \multicolumn{4}{|c|}{ Adenomas 25 cases } \\
\hline normal & 19 & 1 & 1 \\
\hline reduced & - & 12 & 1 \\
\hline weak/absent & - & - & 7 \\
\hline \multicolumn{4}{|c|}{$U C$ dysplasia 8 cases } \\
\hline normal & 1 & - & - \\
\hline reduced & - & - & - \\
\hline weak/absent & - & - & 7 \\
\hline
\end{tabular}



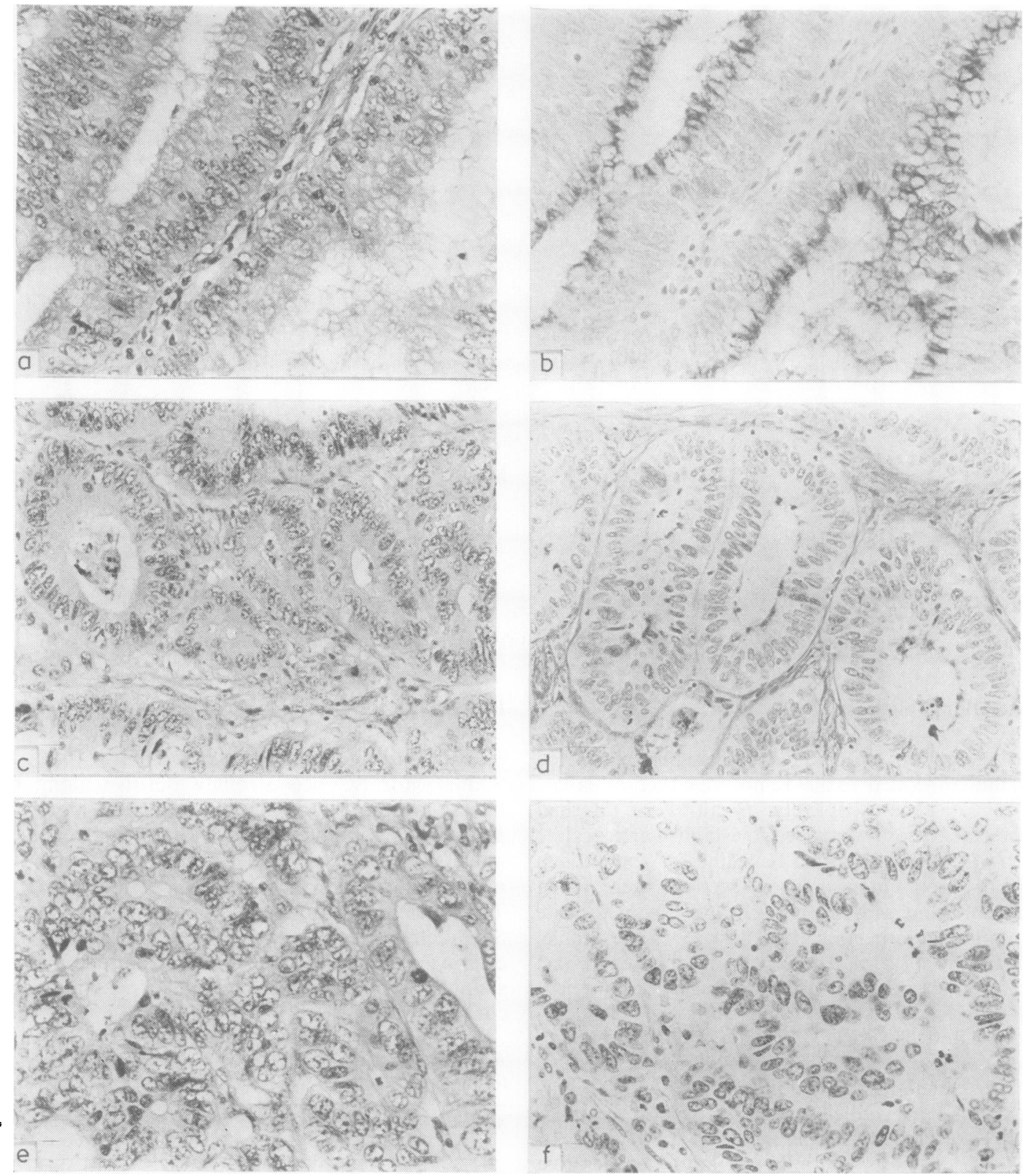

Fig. 2 Representative invasive areas from the same section of a colorectal carcinoma showing (a) well differentiated tumour with (b) strong staining for SC, (c) moderately differentiated tumour with (d) reduced SC staining, and $(e)$ poorly differentiated tumour with $(f)$ absent $S C$ staining. a, $c, e$ : haematoxylin and eosin $\times 200$; $b, d, f$ : immunoperoxidase $\times 200$.

\section{COLORECTAL CARCINOMAS}

The results of staining for SC are summarised in the Table. With the exception of some poorly differentiated tumours which showed uniform absence of SC staining there was marked variation in intensity of SC staining throughout the sections. It was therefore impossible to grade the intensity of SC staining in the section as a whole. With closer scrutiny it became clear that variation in SC staining was regional and closely followed morphological changes 
in both the cytology and architectural arrangement of the tumour cells (Fig. 2). Intensity of staining comparable to normal mucosa was found only in areas of tumour that were well differentiated whilst very weak or absent staining always correlated with poor differentiation. Between these two extremes staining was always reduced in intensity and, although the degree of reduction varied considerably, it again appeared to correlate closely with differentiation. It was not possible, however, to characterise these moderately differentiated foci into reproducible subgroups. Accordingly, representative areas of each of the three degrees of differentiation were sought in each tumour studied and the related intensity of SC staining noted. The reverse procedure of correlating intensity of SC staining with morphological differentiation was also carried out so that for each case studied an assessment was reached correlating intensity of SC staining with each degree of differentiation present. Thus, amongst the 25 cases of carcinoma studied, eight showed well differentiated areas; in six of these SC staining was normal and in two it was reduced in intensity. Poorly differentiated areas were present in 21 cases and in 20 of these staining was very weak or absent, while in one reduced staining was noted. Areas of moderate differentiation were noted in 17 cases and SC staining was reduced in intensity in 16 and normal in one. It was notable that cytological differentiation was the critical factor in determining SC staining rather than the overall architecture of the tumourthat is, gland formation, budding etc, although the two were usually related. With decreasing degrees of differentiation there was increase in nuclear: cytoplasmic ratio, nuclear hyperchromatism and prominence of nucleoli. Stains of the malignant epithelial cells for $\operatorname{IgA}$ and $\mathbf{J}$ chain paralleled SC staining and there was striking correlation between mucosal SC and stromal IgA plasma cells. Thus, foci of decreased or absent SC staining were always accompanied by markedly decreased numbers of IgA-containing plasma cells (Fig. 3).

\section{ADENOMAS}

Here again intensity of SC staining was often not uniform and was dependent on the degree of dysplasia within the adenoma be it tubular, tubulovillous or villous. Adenomas were therefore assessed following the same procedure as that used for carcinomas with correlation of intensity of SC staining with the degree of dysplasia (Table). Dysplasia in the adenomas was classified as mild, moderate, or severe according to the criteria of Morson. ${ }^{8}$ Foci of mild dysplasia in adenomas (Fig. 4) stained strongly for SC while severely dysplastic foci showed very weak or negative staining. Foci of moderate dysplasia showed reduced intensity of staining. Thus the pattern of staining was similar to that in carcinomas and when the cytology was carefully evaluated the different degrees of cytological differentiation paralleled those seen in carcinomas (Figs. 5, 6, 7). The mildly dysplastic cells in adenomas were morphologically similar to those of well differentiated areas in carcinomas and stained similarly for SC. Progressive increase in nuclear enlargement, loss of nuclear polarity and stratification of nuclei in both adenomas and carcinomas was associated with decrease and, finally, loss of SC staining. Staining for IgA and $J$ chain produced results similar to those found in carcinomas with marked reduction of IgA-containing plasma cells in areas where SC was decreased or absent (Fig. 8).

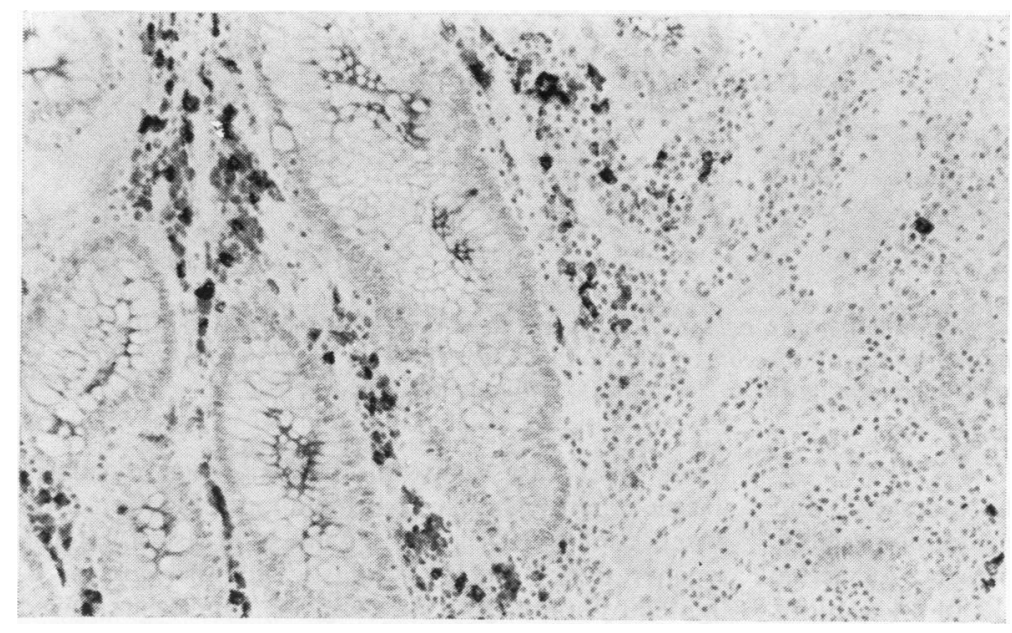

Fig. 3 Section of poorly differentiated carcinoma (right) adjacent to normal colorectal mucosa stained for $\operatorname{Ig} A$. Abundant positively staining plasma cells are present between normal glands but virtually absent within the tumour. Immunoperoxidase $\times 100$. 

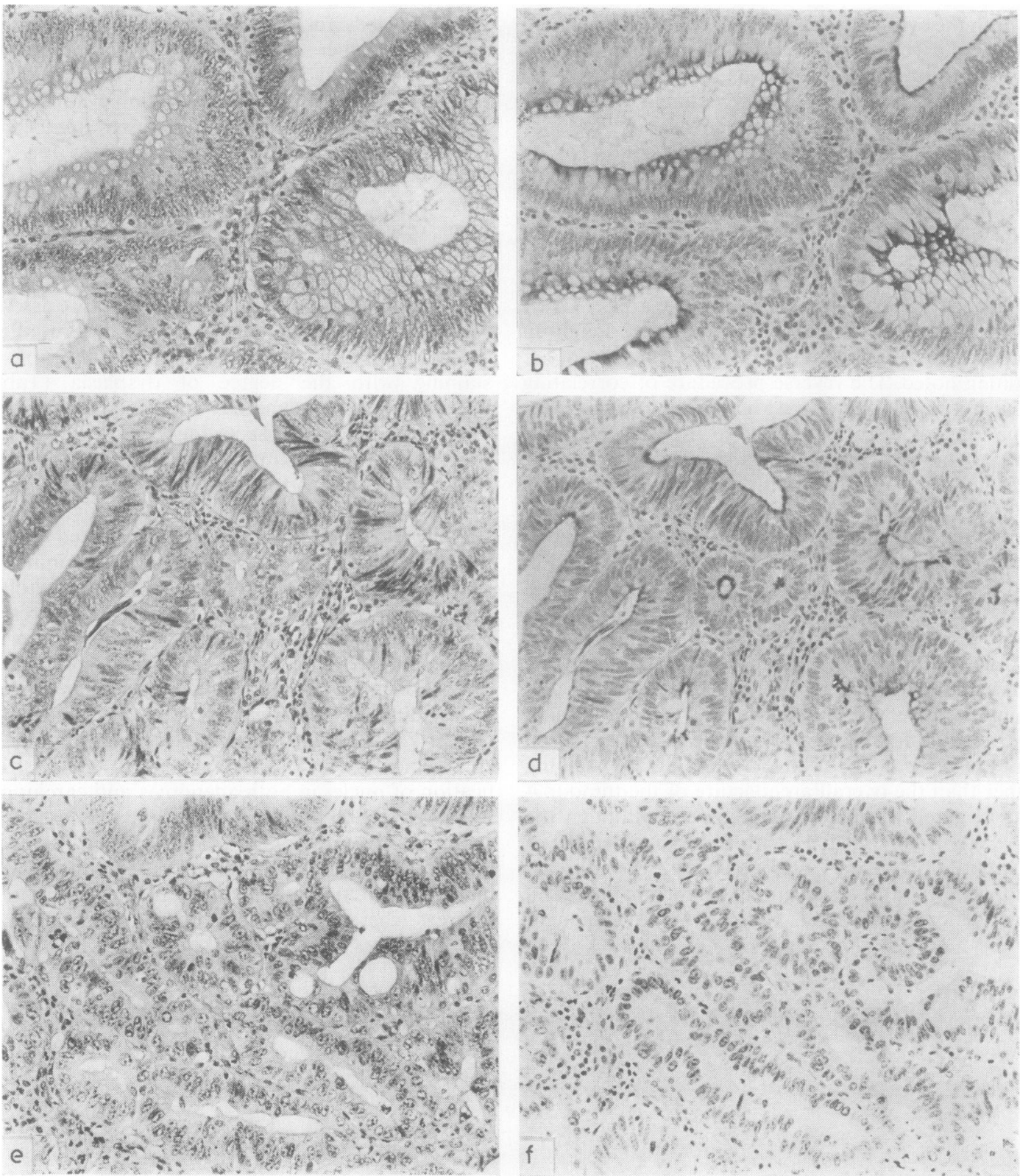

Fig. 4 Representative areas from a single section of a colorectal adenoma showing (a) mild dysplasia with $(b)$ strong $S C$ staining, $(c)$ moderate dysplasia with $(d)$ reduced $S C$ staining and $(e)$ severe dysplasia with $(f)$ absent SC staining. $a, c, e$ : haematoxylin and eosin $\times 200 ; b, d, f$ : immunoperoxidase $\times 200$.

ULCERATIVE COLITIS

Sections were selected to include quiescent and highly active phases of the disease. Staining for SC was strongly positive even in the presence of active mucosal regeneration (Fig. 9). In 13 of 14 sections from eight cases showing precancerous dysplasia (Table) there was marked depletion and often total absence of SC (Fig. 10). Amidst areas of intense regeneration isolated dysplastic glands were highlighted by staining for SC (Fig. 11). One case showed 

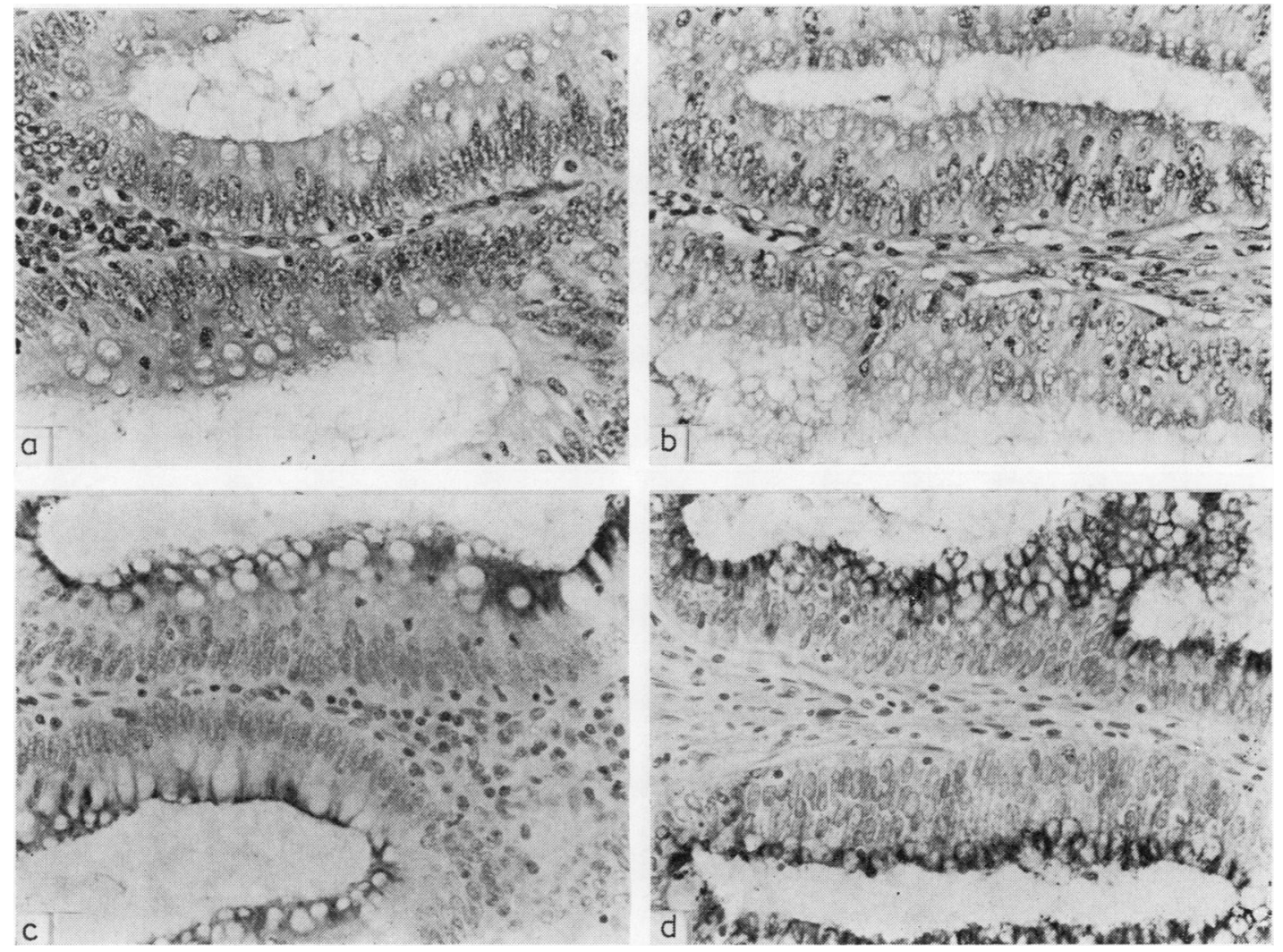

Fig. 5 Comparative histology of (a) mildly dysplastic adenoma and $(b)$ well differentiated carcinoma showing marked similarity. SC staining intensity of $(c)$ adenoma and $(b)$ carcinoma, are also similar. $a, b$ : haematoxylin and eosin $\times 400 ; c, d$ : immunoperoxidase $\times 400$.

normal SC staining and in this case the dysplastic area was macroscopically unusually well defined and histologically bore a close resemblance to an adenoma with mild dysplasia (Fig. 12). Careful cytological evaluation of the other cases showed appearances similar to those in severely dysplastic foci of adenomas and poorly differentiated carcinomas. Some of the sections of dysplasia were characterised by villous processes covered by mucincontaining cells (Fig. 13). The cells at the bases of these villi showed marked cytological dysplasia and stained negatively for SC. This negative staining continued into the mucin-containing cells which, cytologically, showed little or no dysplasia. Staining for IgA and J chain revealed positively staining plasma cells in the inflamed lamina propria of regenerative and dysplastic areas in equal concentration.

\section{Discussion}

The results of this study showing progressive loss of SC synthesis with increasing degrees of dysplasia or dedifferentiation are in agreement with the two previous studies of Poger et $a l^{9}$ and WeiszCarrington et al ${ }^{10}$ both reported from the same laboratory. Using immunofluorescence on frozen sections these workers noted diminished or absent SC synthesis in poorly differentiated colorectal carcinomas and areas of severe dysplasia in adenomas. They did not, however, note the great variability of SC staining and differentiation within the same section of colorectal carcinoma, and each tumour was simply classified as well, moderately, or poorly differentiated. It is clear, from the results of the present study that few colorectal carcinomas are homogeneous in their differentiation. A tumour may 

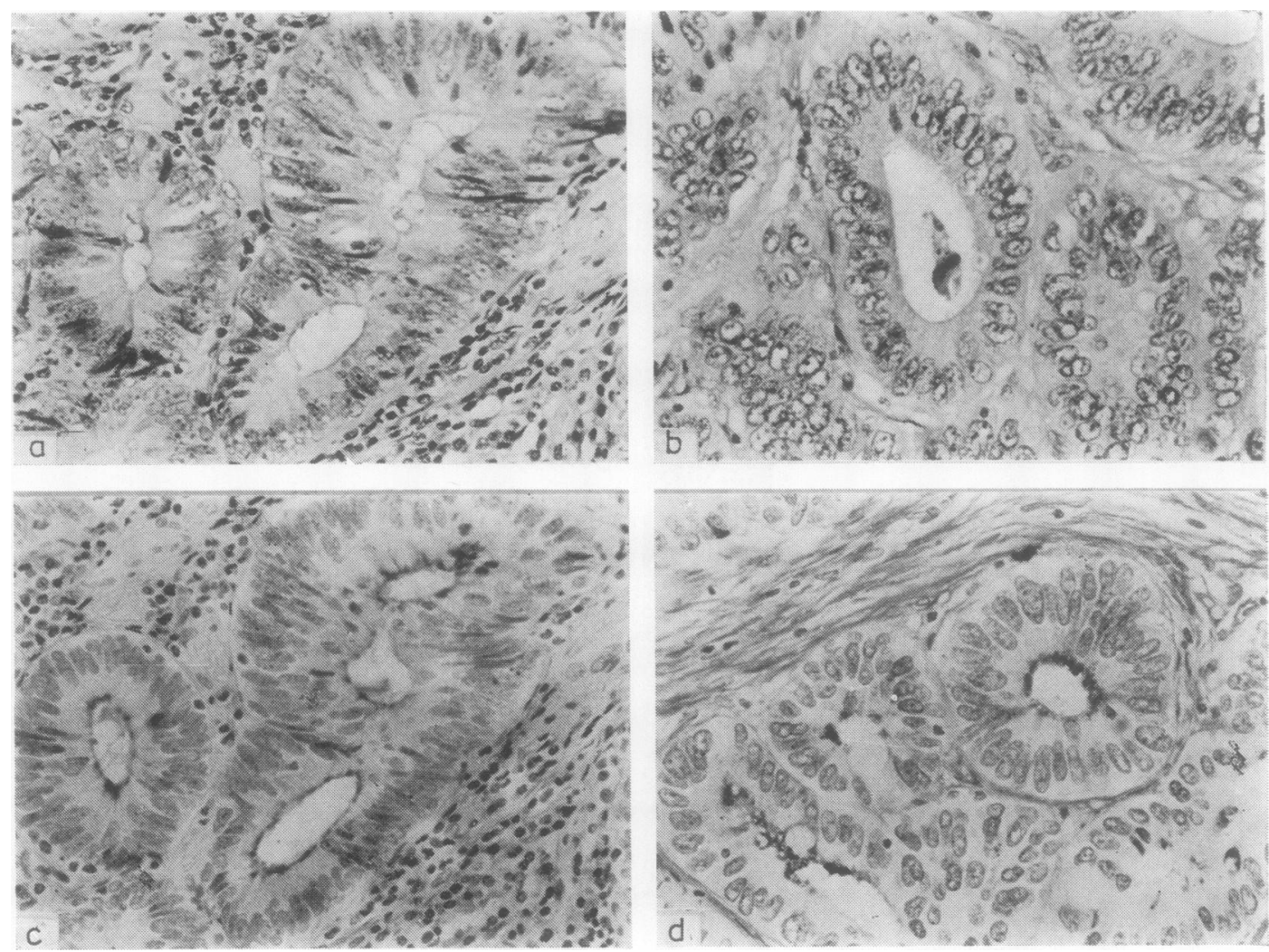

Fig. 6 (a) Moderate dysplasia in an adenoma compared to (b) moderate differentiation in a carcinoma showing similar appearances. SC staining of $(c)$ adenoma and $(d)$ carcinoma, both show similar reduction in intensity. $a, b$ : haematoxylin and eosin $\times 400 ; c, d$ : immunoperoxidase $\times 400$.

superficially appear homogeneous in a routinely stained haematoxylin and eosin section but staining for SC often reveals marked differences in staining intensity between groups of cells which on closer inspection are related to cytological differences. Studies relating differentiation of colorectal cancers to prognosis have not taken this lack of homogeneity into account and their conclusions must be open to doubt.

The results of staining adenomas for SC are of great interest. The gradual loss of SC paralleling cytological (and usually architectural) dedifferentiation reinforces the validity of Morson's concept that dysplasia is a spectrum of changes characterised by graded degrees within adenomas. ${ }^{8}$ At first sight, however, it is difficult to reconcile absent SC staining in severely dysplastic foci of adenomas with positive staining in many carcinomas since the accepted view is that adenomas pass through increasingly severe grades of dysplasia before transforming into carci- nomas. This paradox is also apparent in the results of the studies of Poger et al and Weisz-Carrington et al neither of whom commented on it, however. It can be explained by close examination of cytological detail which shows that positively staining areas in carcinomas are cytologically markedly similar to those in adenomas as are areas showing reduced and weak to absent staining. Thus, it would seem that, at the cytological level, differentiation in adenomas and carcinomas is similar and that the terms dysplasia and differentiation are really synonymous. Morson's concept of dysplasia as a continous spectrum of changes in adenomas can, therefore, be extended to include invasive lesions. Does this mean that severe dysplasia in adenomas is of no account? A glance at the Table will show few well differentiated-that is, mildly dysplastic, foci within carcinomas and likewise few severely dysplastic - that is, poorly differentiated areas in adenomas. It should be stressed too, that well differentiated foci in carcino- 

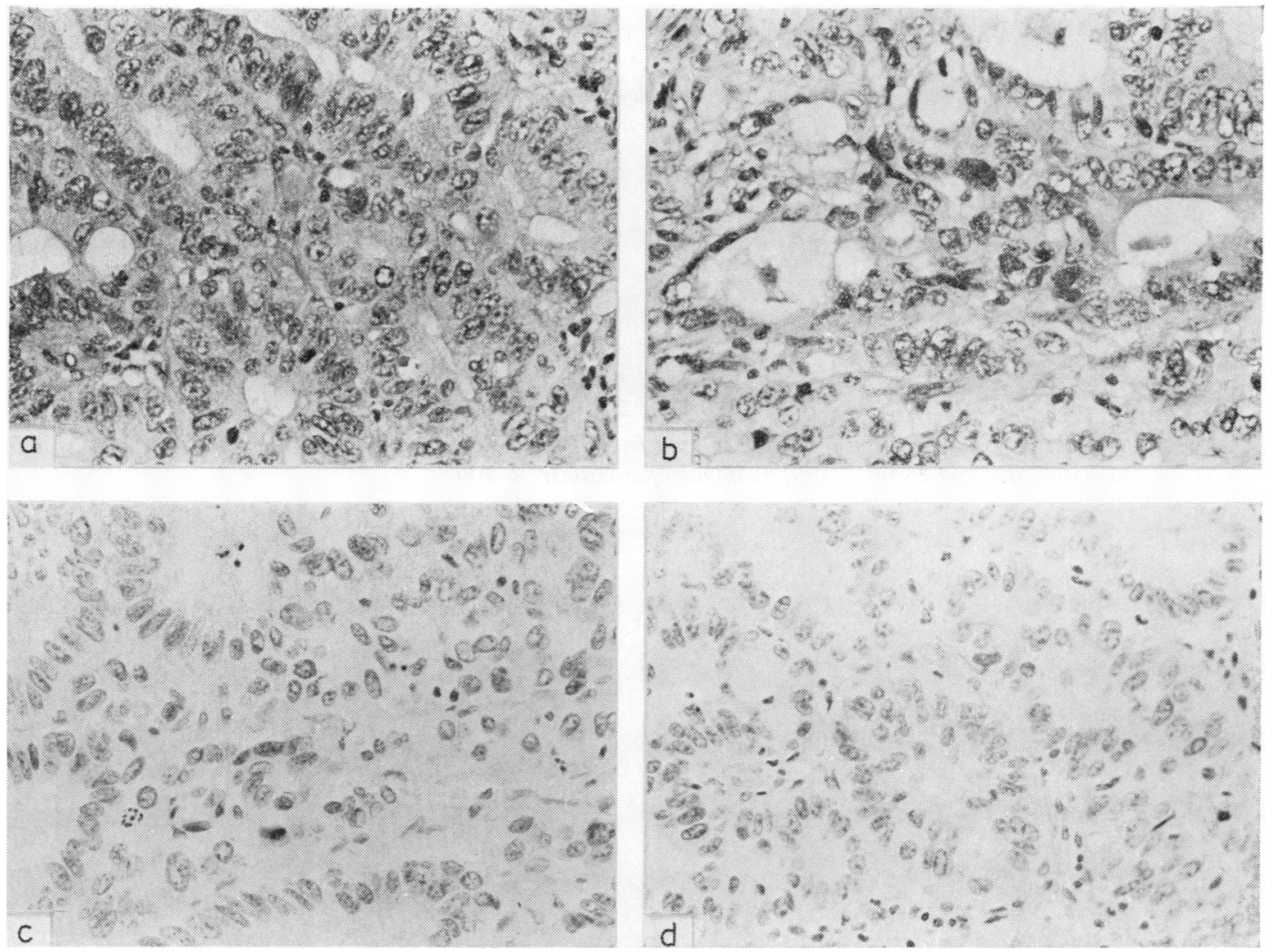

Fig. 7 (a) Severe dysplasia in an adenoma showing similar appearances to (b) poorly differentiated carcinoma. There is absence of SC staining in both (c) the adenoma and $(d)$ the carcinoma. $a, b$ : haematoxylin and eosin $\times 400 ; c, d$ : immunoperoxidase $\times 400$.

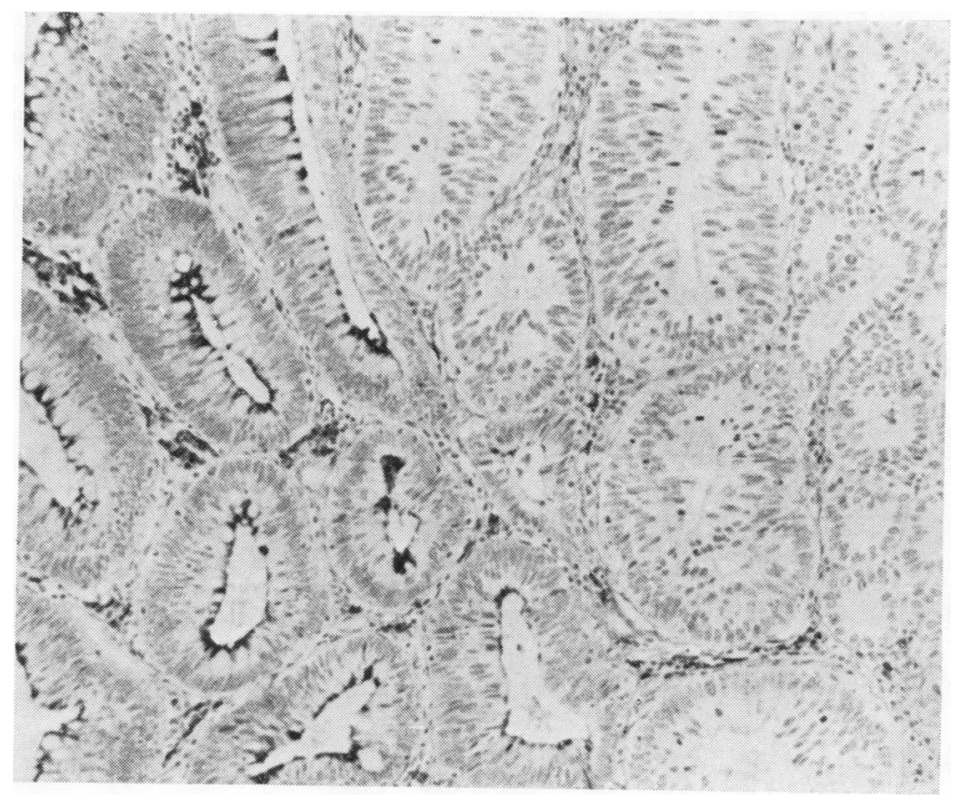

Fig. 8 A section of an adenoma (same case as Fig. 4) stained for $\operatorname{Ig} A$. There is strong staining of secretory IgA and plasma cells in the mildly dysplastic area on the left with only a few plasma cells present in the severely dysplastic area on the right. Immunoperoxidase $\times 100$. 

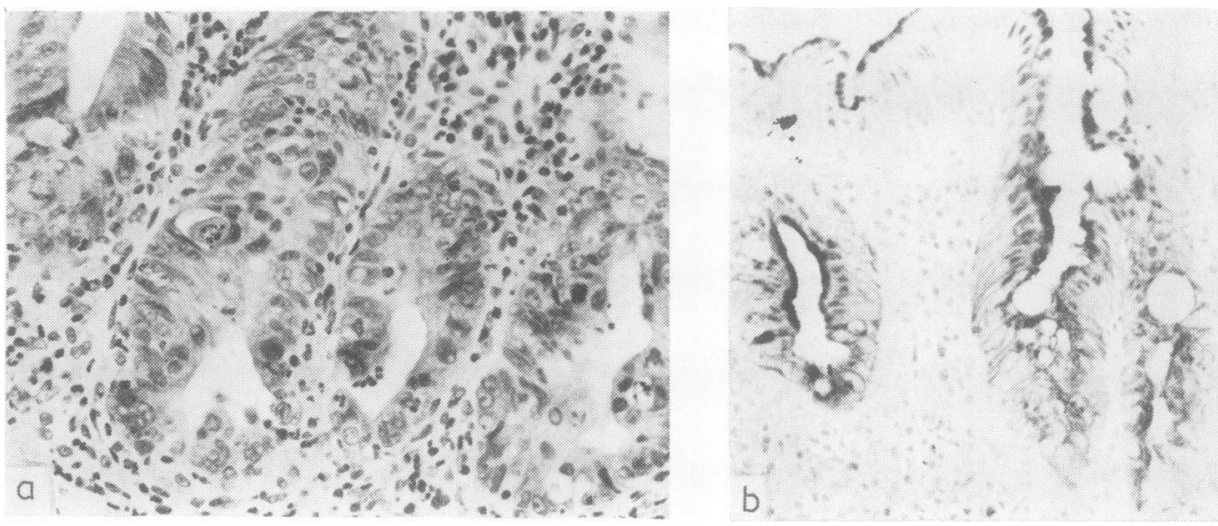

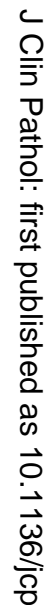

Fig. 9 (a) Actively regenerating inflamed mucosa from a case of ulcerative colitis showing (b) strong staining for $S C$. a: haematoxylin and eosin $\times 100 ; b$ : immunoperoxidase $\times 100$.
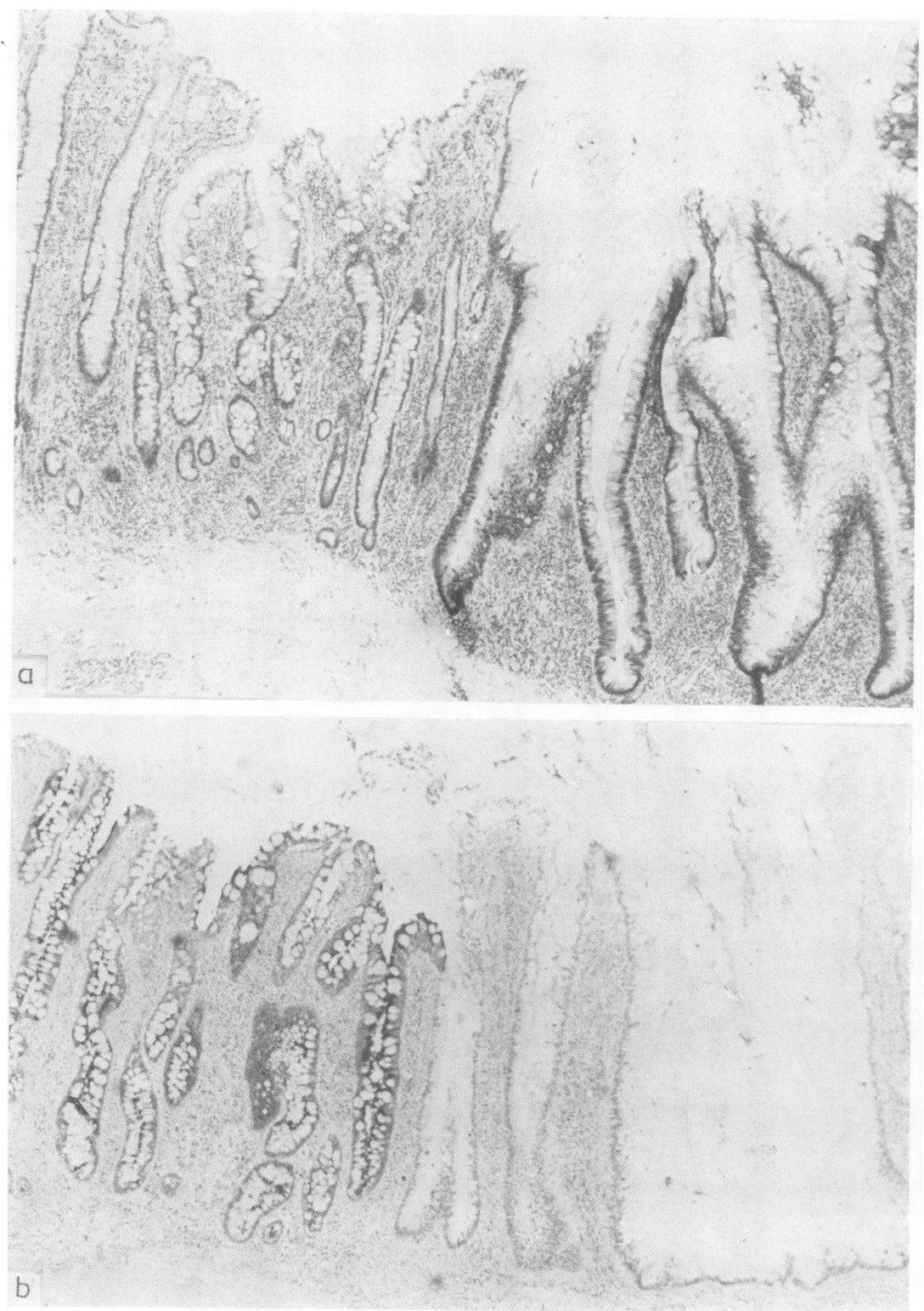

Fig. $10 \quad$ (a) $A$ case of ulcerative colitis showing precancerous dysplasia ono the right. (b) Same field stained for SC showing absent staining in dysplastic mucosa. a: haematoxylin and eosin $\times 40 ; b$ : immunoperoxidase $\times 40$ 을 

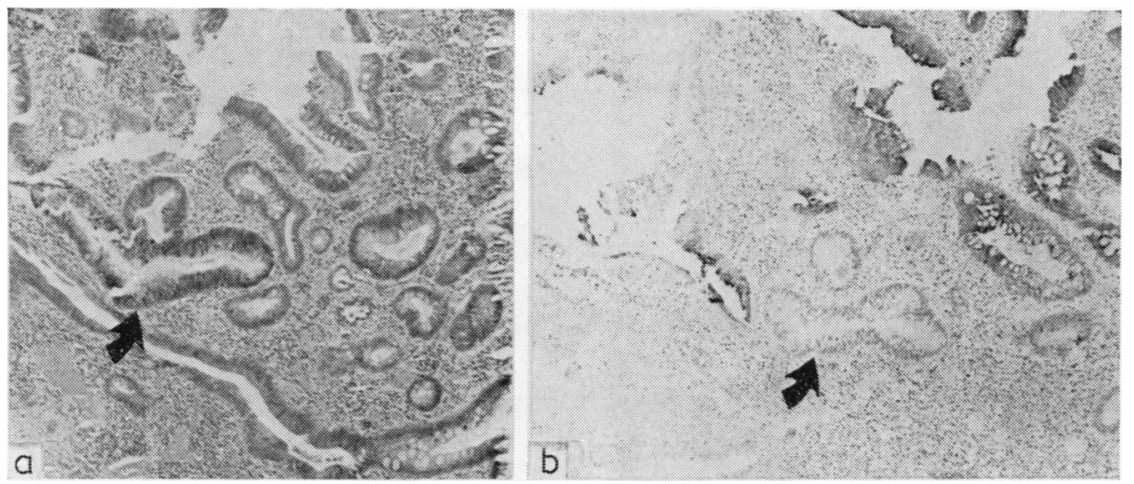

Fig. 11 (a) Active ulcerative colitis with a focus of dysplasia (arrow). (b) Same field stained for SC which highlights the SC negative dysplastic focus in contrast to surrounding positively staining inflamed mucosa. a: haematoxylin and eosin $\times 40 ; b$ : immunoperoxidase $\times 40$.
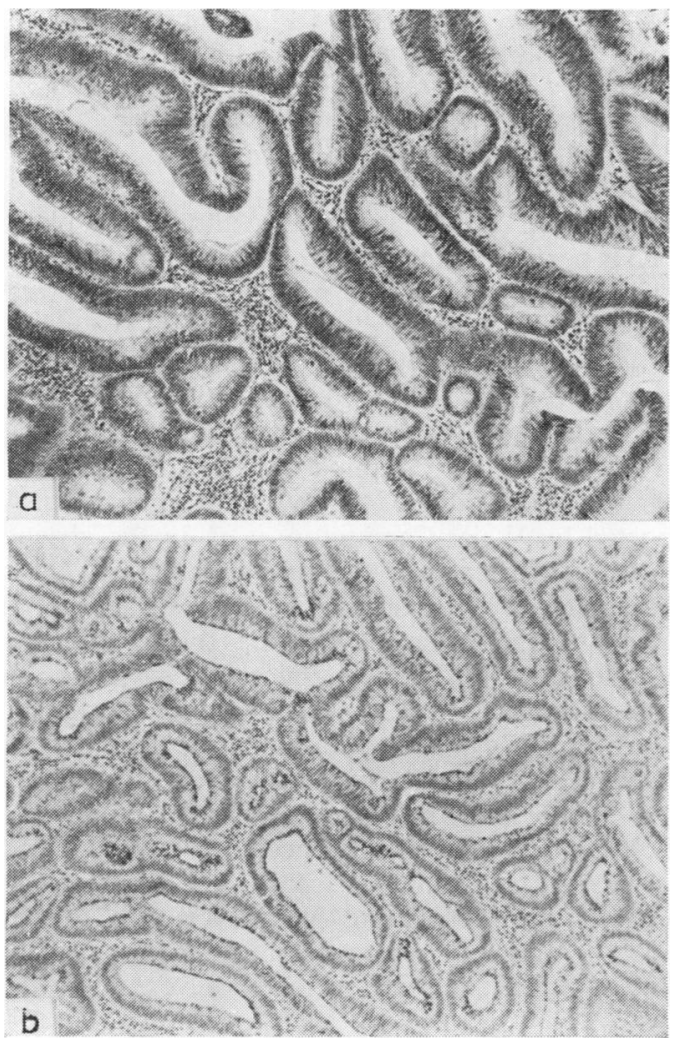

Fig. 12 (a) A focus of dysplasia from a case of ulcerative colitis resembling a mildly dysplastic adenoma. (b) SC staining showing normal intensity.

$a$ : haematoxylin and eosin $\times 100 ; b$ : immunoperoxidase $\times 100$. mas were usually small in relation to the whole section; likewise the severely dysplastic foci in adenomas. There is thus a greater tendency for severely dysplastic foci to become invasive but severe dysplasia is not a necessary precursor of invasion. Most pathologists will be familiar with examples of well or moderately differentiated invasive carcinoma arising in large adenomas which may not show severe dysplasia. Thus, in terms of SC synthesis and morphology there is no difference between cells comprising colorectal adenomas and carcinomas and this suggests that the important switch in the progression towards invasive malignancy occurs at the level of the adenoma. Whether invasiveness requires a second fundamental change in the cell or is simply a matter of time is uncertain.

When applied to examples of precancerous dysplasia in ulcerative colitis the results of SC staining are often dramatic but not unexpected. The dysplasia in these cases is usually severe and correspondingly the invasive carcinomas that arise in UC are usually poorly differentiated. In five of the eight cases of precancerous dysplasia included in this study there was an associated poorly differentiated carcinoma. All of these, which were not included in the 25 cases of carcinoma described above were poorly differentiated and stained negatively for SC. Again, to appreciate the severity of the dysplasia, it is the assessment of the cytology of individual cells that is important. The lack of SC staining in the mucinous columnar cells covering villous processes which characterise some cases of precancerous dysplasia suggests that these cells are severely dysplastic although their morphological characteristics do not indicate this. The cells at the bases 

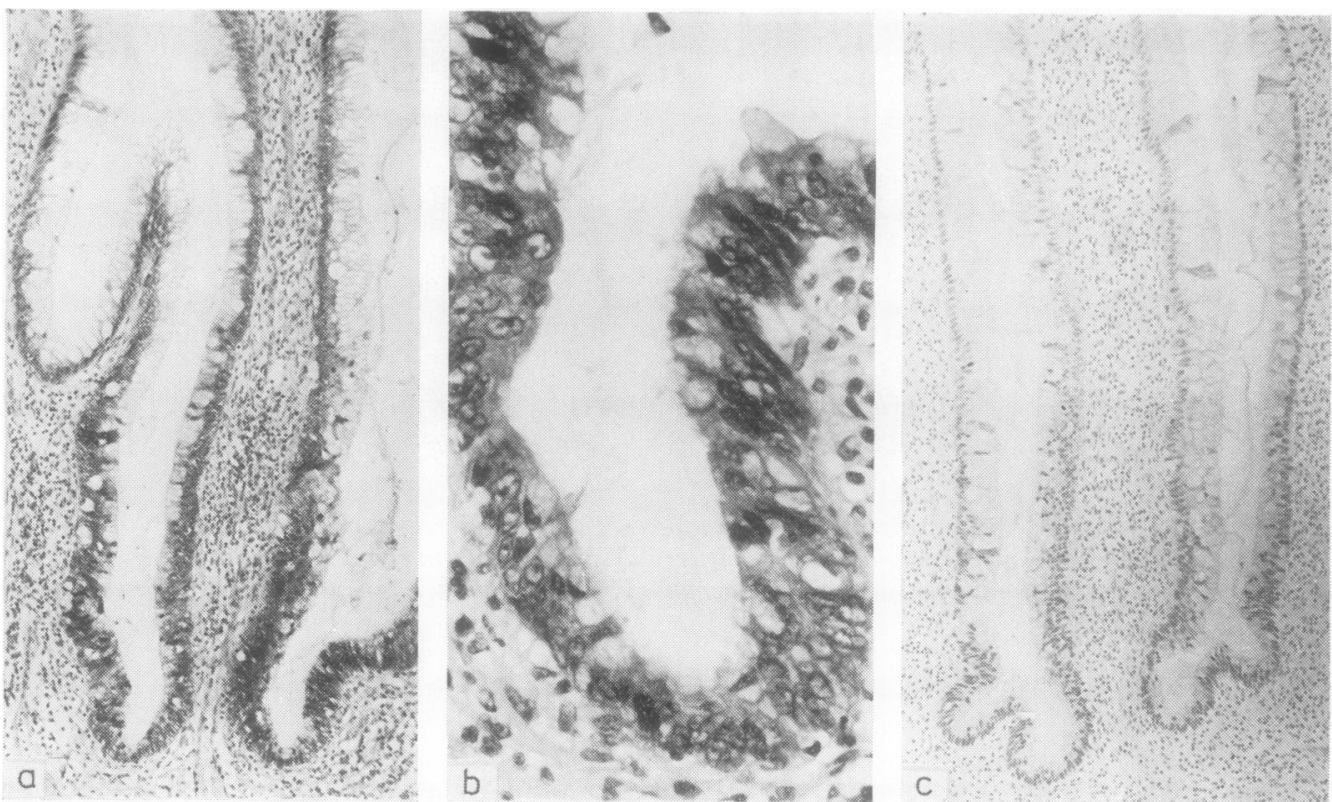

Fig. 13 (a) Precancerous dysplasia from a case of UC showing maturation of mucus-containing cells along villous structures. (b) High power view of a base of a dysplastic crypt showing severe dysplasia. (c) Same field stained for SC showing absence of staining in the bases of the crypts and in mucus cells along the villous structures. $a, b$ : haematoxylin and eosin $\times 100$ and $\times 400 ; c$ : immunoperoxidase $\times 100$.

of these villi appear to differentiate morphologically but not functionally as they progress up the villous stalks. Poger et al thought that they could relate SC synthesis to the presence of mucin but as emphasised by the findings in precancerous dysplasia this study does not confirm this.

The question naturally arises as to the diagnostic value of staining for SC to detect precancerous dysplasia. In active ulcerative colitis differentiation between regeneration and precancerous dysplasia can be extremely difficult. Some success in differentiating these two conditions can be achieved by staining immunohistochemically for CEA. ${ }^{11}$ This rests, however, on careful adjustments of the titre of the antiserum to achieve a quantitative rather than qualitative distinction between CEA synthesis in the two conditions and this, coupled with the variation in specificity of different antiCEA sera, meant that the technique was not suitable for routine use. Provided that antiSC serum is titred out to give crisp specific staining on normal colon there is little effect on differential staining between normal and dysplastic mucosa by a shift in titre of one or two dilutions in either direction and antisera from two different sources and species have given identical results. Foci of mild dysplasia which bear a strong resemblance to mildly dysplastic adenomas as occurred in one case in this study will not be distinguished by SC staining. Although dysplasia, when it occurs in ulcerative colitis, tends to be severe distinction from inflamed regenerative mucosa can still be difficult, and here SC staining could be helpful as shown in Fig. 11. Further prospective studies on biopsy specimens are needed to fully evaluate the usefulness of SC staining as a diagnostic tool.

The relation of lamina propria IgA plasma cells to the SC status of the mucosa is intriguing. As reported by Weisz-Carrington et al, in the absence of overt inflammation there is an extremely close relationship between absence of SC and absence of IgA-containing plasma cells and this relationship holds true within a single microscopic field. This suggests a role for SC in the homing mechanism of IgA lymphocytes to the lamina propria of the gut; a hypothesis originally put forward by Guy-Grand et $a l^{12}$ but later refuted by Brandtzaeg et al. ${ }^{13}$ Alternatively, IgA may be necessary to induce SC production by mucosal cells. The presence of abundant $\operatorname{IgA-containing~plasma~cells~in~the~SC~}$ negative inflamed dysplastic mucosa of ulcerative colitis is against this suggestion however.

\section{References}

${ }^{1}$ Isaacson P, Le Vann HP. The demonstration of carcinoembryonic antigen in colorectal carcinoma and colonic 
poiyps using an immunoperoxidase technique. Cancer 1976;38:1348-56.

2 Isaacson P, Judd MA. Immunohistochemistry of carcinoembryonic antigen in the small intestine. Cancer 1978; 42:1554-9.

${ }^{3}$ Neville AM, Laurence DJR. Tumour markers and the gastrointestinal tract. In: Wright $\mathrm{R}$, ed. Recent advances in gastrointestinal pathology. London: WB Saunders, 1980:255-66.

4 Brown WR. Relationships between immunoglobulins and the intestinal epithelium. Gastroenterology 1978;75:12938.

${ }^{5}$ Mepham BL, Frater W, Mitchell BS. The use of proteolytic enzymes to improve immunoglobulin staining by the PAP technique. Histochem $J$ 1979;11:345-57.

${ }^{6}$ Isaacson $\mathrm{P}$. Immunochemical demonstration of $\mathrm{J}$ chain: a marker of B-cell malignancy. J Clin Pathol 1979;32: 802-7.

7 Isaacson P. Crypt cell carcinoma of the appendix-socalled adenocarcinoid tumour. Am J Surg Pathol 1981; 5:213-24.

${ }^{8}$ Morson BC, Konishi F. Dysplasia in the colorectum. In: Wright $\mathrm{R}$, ed. Recent advances in gastrointestinal pathology. London: WB Saunders, 1980:331-43.

${ }^{9}$ Poger ME, Hirsch BR, Lamm ME. Synthesis of secretory component by colonic neoplasms. Am J Pathol 1976; 82:327-34.

${ }^{10}$ Weisz-Carrington P, Poger ME, Lamm ME. Secretory immunoglobulins in colonic neoplasms. Am J Pathol 1976;85:303-14.

"Isaacson P. Tissue demonstration of carcinoembryonic antigen (CEA) in ulcerative colitis. Gut 1976;17:561-7.

${ }^{12}$ Guy-Grand D, Griscelli C, Vassalli P. Gut-associated lymphoblasts and intestinal IgA plasma cells. $A d v$ Exp Med Biol 1974;45:41.

${ }^{13}$ Brandtzaeg $\mathrm{P}$, Gjeruldsen ST, Korsrud F, Baklien $\mathrm{K}$, Berdal P, Ek J. The human secretory immune system shows striking heterogeneity with regard to involvement of J chain-positive IgD immunocytes. J Immunol 1979; 122:503-10.

Requests for reprints to: Dr P Isaacson, Department of Pathology, Level E, South Laboratory Block, General Hospital, Southampton SO9 4XY, England. 